REVIEW

\title{
My approach to serrated polyps of the colorectum
}

\author{
T Higuchi, J R Jass
}

J Clin Pathol 2004;57:682-686. doi: 10.1136/icp.2003.015230

Hyperplastic polyps of the colorectum are heterogeneous lesions, a subset of which is now regarded as the precursor of colorectal cancer with DNA microsatellite instability. Some authors have distinguished this subset from classic hyperplastic polyps and have introduced the term "sessile serrated adenoma". These lesions frequently show BRAF mutation and DNA methylation. This personal perspective reviews recent insights into serrated polyps and highlights the importance of inhibition of apoptosis as a unifying mechanism. It is estimated that around 25 hyperplastic polyps of the proximal colon exist for every colorectal cancer with DNA microsatellite instability. Further research is required to identify additional risk factors for hyperplastic polyps other than anatomical location. These may be demographic, clinical, morphological, or molecular. It is not recommended that the term sessile serrated adenoma be used in routine reporting, but it is desirable that potentially aggressive hyperplastic polyps should be identified for the purposes of both clinical practice and research.

See end of article for authors' affiliations

Correspondence to: Professor J R Jass, Department of Pathology, McGill University, Duff Medical Building, 3775 University Street, Montreal, Quebec, Canada H3A 2B4; jeremy.jass@ mcgill.ca

Accepted for publication 12 January 2004
D uring the past few years, it has become clear that hyperplastic polyps of the colorectum can no longer be dismissed as "non-neoplastic lesions with no malignant potential". The basis for this premise has been published in pathological, ${ }^{1-3}$ clinical, $^{45}$ and basic science $^{6}$ literature, and has been incorporated into standard texts, ${ }^{7}$ including the most recent World Health Organisation (WHO) classification of tumours of the digestive system. ${ }^{8}$ This new position brings with it several problems that need to be resolved. First, the fact that a statement is true and has been widely published does not mean that it is has been generally accepted and incorporated within routine clinical practice. Nevertheless, it is expected that scientific evidence will ultimately triumph over dogma with time. Second, as is the case for colorectal adenomas, it is clear that most hyperplastic polyps are clinically unimportant. That is, if left alone, most would not progress to cancer. Hyperplastic polyps in the distal colorectum apparently do not predict the presence of clinically important pathology in the proximal colon, and neither do they predict the development of significant lesions in the future. ${ }^{9}$ However, as is the case with colorectal adenomas, there is evidence that certain types of hyperplastic polyp are linked with colorectal neoplasia. On this basis, the third problem centres upon how we recognise these polyps and introduce the term that should be used for naming these high risk polyps in reports to clinicians. The issue of nomenclature is by no means trivial and settling on internationally approved terms and definitions for pathological lesions can take many years of rancorous debate. The current WHO classification includes reference to hyperplastic polyp, mixed hyperplastic polyp/adenoma, and serrated adenoma. ${ }^{8}$ There is no existing customised label for a particular type of hyperplastic polyp that may be of clinical concern.

\section{THE CONCEPT OF "SERRATED POLYPS"}

The term "serrated polyp" was introduced to conceptualise the relatedness of hyperplastic polyps and serrated adenomas. ${ }^{10}$ Before this point, serrated adenomas were conceived as variant adenomas and grouped with adenomas as bona fide neoplasms. ${ }^{11}$ The WHO classification continues to group serrated adenoma with adenomas and to retain hyperplastic polyps as a separate category. ${ }^{8}$ Nevertheless, mixed polyps frequently represent the combination of hyperplastic polyp and serrated adenoma. ${ }^{2}$ In addition, serrated adenomas and hyperplastic polyps have three key molecular features in common. First, they both show mutation of genes in the mitogen activated protein kinase signalling pathway: BRAF or K-ras. ${ }^{12}{ }^{13}$ Second, they both show extensive DNA methylation. ${ }^{14-16}$ Third, they both show gastric metaplasia. ${ }^{517}$ The "serrated pathway" of colorectal neoplasia has been linked with a particular subtype of sporadic colorectal cancer that is also characterised by DNA methylation, mutation of either BRAF or K-ras, and gastric metaplasia. ${ }^{18-20}$ In addition, these cancers usually show DNA microsatellite instability to a high (MSI-H) or low degree. ${ }^{18-20}$ The morphological features of classic serrated adenomas have been reviewed in detail elsewhere. ${ }^{21}$ Briefly, serrated adenomas differ from classic hyperplastic polyps in showing unequivocal epithelial dysplasia, increased architectural complexity, pronounced cytoplasmic eosinophilia, absence of thickening of the basement membrane underlying the surface epithelium, and a relative lack of endocrine cells. ${ }^{11} 22$

"The hyperplastic polyp can be defined as a
lesion caused by the inhibition of pro-
grammed cell death or apoptosis"

Abbreviations: $\mathrm{MSI}$, microsatellite instability; $\mathrm{MSI}-\mathrm{H}$, high degree of microsatellite instability; MUHC, McGill University Health Centre; WHO, World Health

Organisation 
Despite the fact that it is logical to group together hyperplastic polyps and serrated adenomas as "serrated polyps", this manoeuvre runs the risk of over emphasising the importance of serration. Serration can be seen in a variety of contexts outside of hyperplastic polyps and serrated adenomas. These include solitary rectal ulcer syndrome (particularly polypoid variants), the hyperplastic mucosa adjacent to colorectal cancer, chronic inflammatory bowel disease, juvenile polyps, and colorectal cancer. In addition, the borderline between serrated adenoma and conventional adenoma (particularly when tubulovillous or villous) is blurred. Rather, the focus should be on the molecular mechanisms that underlie the serrated pathway. It has been known for years that hyperplastic polyps are characterised by the abnormal retention of surface epithelium..$^{23}$ Using more modern terminology, the hyperplastic polyp can be defined as a lesion caused by the inhibition of programmed cell death or apoptosis. This is likely to be a form of apoptosis peculiar to epithelial surfaces that is initiated by programmed exfoliation of surface epithelial cells. This has been described as "anoikis". ${ }^{25}$

Inhibition of anoikis will be the underlying mechanism common to all lesions showing epithelial serration. One can conceive of two broad mechanisms that could lead to the inhibition of anoikis. One would be epigenetic and in response to tissue injury, whether this be inflammatory or ischaemic. The second would be genetic or "neoplasia-like", insofar as the mechanism involves mutation of a protooncogene, such as BRAF or K-ras. For example, mutation of $\mathrm{K}$-ras has been linked to the inhibition of apoptosis through Akt/protein kinase $\mathrm{B}$, which inhibits caspase- $9^{26}$ and $\mathrm{BAD},{ }^{27}$ and through downregulation of the cell membrane apoptosis receptor Fas or CD95. ${ }^{28}$ Downregulation of Fas has been demonstrated in serrated adenomas. ${ }^{29}$

Inhibition of apoptosis characterises serrated polyps, whereas loss of inhibition of proliferation is the mechanism underlying tubular adenomas. These are two sides of the same coin in that they both result in increased epithelial growth. Both mechanisms are integral to tumorigenesis. Inhibition of apoptosis has the particular attribute of facilitating the development of genetic instability, which may be defined as the progressive, non-lethal accumulation of genetic errors. ${ }^{18}$ Serration arises when cells continue to be generated within the proliferative compartment but are blocked from ascending to their rightful location within the epithelial surface. However, serration and hyperplasia are not the only tissue alterations that would result from the inhibition of apoptosis. Other changes consequent on the pressure of increased cell numbers will include crypt dilatation, horizontal growth of crypts, pseudoinvasion, and crypt fission.

\section{RECOGNITION OF AGGRESSIVE HYPERPLASTIC POLYPS}

Over the years, colorectal cancer has been linked to hyperplastic polyps that are large and/or multiple and/or located in the proximal colon. ${ }^{30}$ Multiplicity may equate with the condition hyperplastic polyposis. This term does not refer to the clustering by small hyperplastic polyps in the mucosa surrounding cancer of the distal colorectum. It refers to (a) the presence of at least 30 pan-colorectal hyperplastic polyps, or (b) at least five hyperplastic polyps proximal to the sigmoid colon of which at least two are greater than $10 \mathrm{~mm}$ in diameter, or (c) any number of hyperplastic polyps in a patient who has a first degree relative with hyperplastic polyposis. ${ }^{31}$ Because hyperplastic polyps may evolve into mixed polyps or serrated adenomas, it would seem reasonable to count all advanced serrated polyps for the purposes of meeting the definition of hyperplastic polyposis. In addition, polyps removed over time may be used to provide a cumulative total on the basis that most of these are likely to be new rather than recurrent polyps. The increased risk of malignancy in association with multiple hyperplastic polyps may not be the result merely of the increased volume of target tissue. Multiple polyps may serve as a marker of a constitutional disturbance in DNA methylation homeostasis, as indicated by the association between DNA methylation in colorectal cancer and a positive family history of cancer. ${ }^{32} \mathrm{~A}$ genetic predisposition to DNA methylation may increase the likelihood of methylating a rate limiting gene, such as hMLHI.

\section{"Polyps removed over time may be used to provide a cumulative total on the basis that most of these are likely to be new rather than recurrent polyps"}

A distinct morphological subtype of colorectal hyperplastic polyp was first highlighted in detail by Torlakovic and Snover in the context of hyperplastic polyposis. ${ }^{22}$ These polyps were characterised by large size, exaggerated serration, crypt dilatation, horizontal crypts, absence of thickened subepithelial collagen plate, increased mucin secretion, hyperproliferation, and absence of endocrine cells (figs 1 and 2). Despite their close resemblance to hyperplastic polyps, it was argued that these lesions were neoplastic and should be regarded as serrated adenomas. The same lesions were subsequently shown to occur sporadically and accounted for around $18 \%$ of all serrated polyps. ${ }^{33}$ They differed from traditional hyperplastic polyps because they could occur in the proximal colon and were termed "sessile serrated adenomas".33 Goldstein et al identified 9l patients with MSI-H colorectal cancer in whom one or more hyperplastic polyps had been diagnosed previously in the same segment of the proximal colon. ${ }^{34}$ The total of 106 hyperplastic polyps were matched with 106 hyperplastic polyps obtained from the distal colorectum and from patients with no history of colorectal cancer. All the study group polyps showed the features of sessile serrated adenoma, whereas this was seen in only $23 \%$ of the control group polyps. ${ }^{34}$ When revisiting our own collection of slides and published photographs of serrated polyps associated with MSI-H colorectal cancer, it is apparent that most show the features of sessile serrated adenoma.

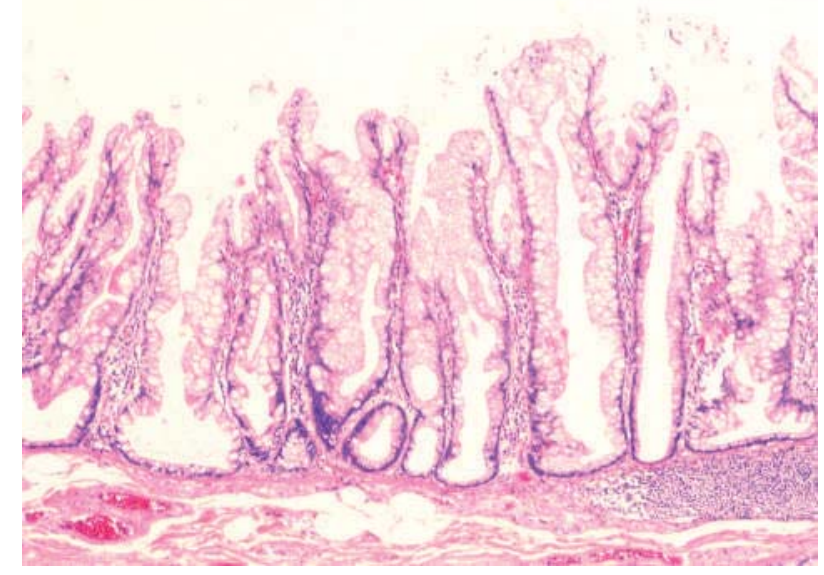

Figure 1 Low power view of a variant hyperplastic polyp described as sessile serrated adenoma showing exaggerated serration within the lower crypt compartment, crypt branching, hypermucinous appearance of the epithelium, and horizontal extension of the crypt base along the muscularis mucosae (haematoxylin and eosin stain). 


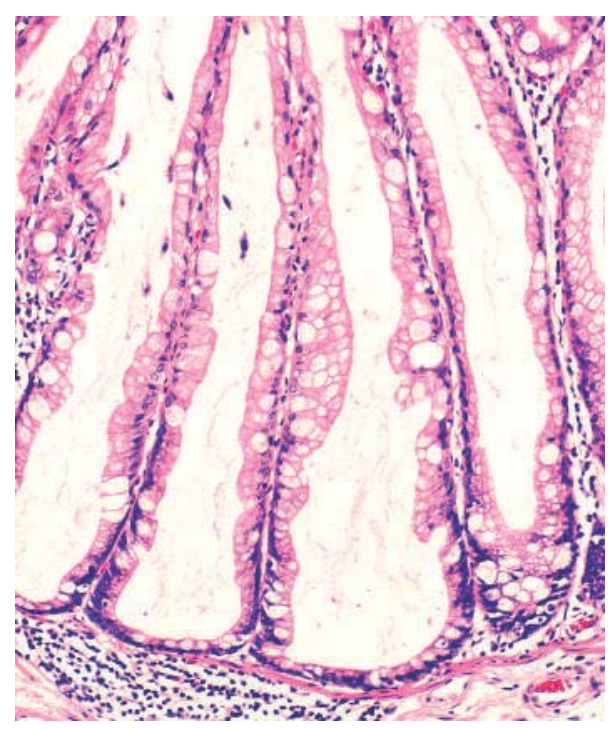

Figure 2 High power view of extremely dilated crypts and relative absence of lamina propria in a sessile serrated adenoma (haematoxylin and eosin stain).

\section{NOMENCLATURE FOR AGGRESSIVE HYPERPLASTIC POLYPS}

Many classic serrated adenomas are sessile. Therefore, the term "sessile serrated adenoma" may be criticised for being too similar to classic serrated adenoma. In addition, most pathologists would interpret many of the illustrations of sessile serrated adenomas in the articles mentioned above as large hyperplastic polyps. For this reason, the alternative term "sessile serrated polyp" was suggested. ${ }^{35}$ Although this adequately describes these lesions, it could be argued that the same term describes the entire spectrum of sessile serrated polyps from classic hyperplastic polyp through to serrated adenoma. It may be questioned whether the sessile serrated adenomas of Torlakovic and colleagues ${ }^{33}$ and Goldstein and colleagues $^{34}$ are fundamentally different at the biological level from classic hyperplastic polyps. Both lesions will arise through inhibition of apoptosis. However, the specific mechanisms underlying apoptosis inhibition may differ and certain mechanisms may result in a more profound tissue disturbance among the sessile serrated adenomas. For example, oncogenic BRAF appears to inhibit caspases at a point downstream of BAD mediated release of cytochrome c from mitochondria. ${ }^{36}$ BRAF mutation and DNA methylation are closely correlated with sporadic MSI-H colorectal cancer. ${ }^{19} 20$ The same changes occur more frequently in sessile serrated adenomas than in classis hyperplastic polyps. ${ }^{16}{ }^{37}$

If there are aggressive hyperplastic polyps then it is desirable that these are recognised and highlighted in reports to the clinician. There is the issue of increased malignant potential and lesions that are considered to be premalignant must be fully excised. There is also the issue of polyps serving as biomarkers for increased risk of metachronous lesions and therefore the introduction of colonoscopic surveillance. The hope of finding a single reliable marker for aggressive hyperplastic polyps may not be realised for some time. Despite decades of research into the behaviour of adenomas, we still do not know which adenomas are aggressive. Yet, there is a principle that should be followed. One first decides upon a clinically important endpoint such as malignant change or prediction of significant remote pathology in terms of type, time, and location. Then one identifies biomarkers that independently predict the endpoints of concern. These biomarkers could be demographic (for example, race, sex, or age), clinical (for example, family history, smoking, or anatomical site), pathological (for example, size, multiplicity, or dysplasia), or molecular (for example, mutation of BRAF or DNA methylation). Different sets of biomarkers may be required to predict different clinical endpoints.

"Despite decades of research into the behaviour of adenomas, we still do not know which adenomas are aggressive"

At this point in time we do not know which features would serve as reproducible and independent markers of high risk hyperplastic polyps, and neither is there an agreed nomenclature for high risk hyperplastic polyps. In the USA, only the diagnosis of adenoma triggers funded follow up surveillance procedures. Perhaps the need to communicate an appropriate level of clinical surveillance influenced the decision to use the term sessile serrated adenoma. ${ }^{34}$ Although expedient, this is not the most scientific rationale for ascribing terminology. To progress this issue, a clinical imperative is required. The first step will be the general acceptance that the status quo regarding hyperplastic polyps is wrong. This point of assent is yet to be realised.

\section{MAGNITUDE OF RISK OF MALIGNANT CHANGE WITHIN HYPERPLASTIC POLYPS}

Although it is likely that hyperplastic polyps from the proximal colon are linked histogenetically with the subset of colorectal cancers showing MSI-H and/or DNA hypermethylation, it is clear that most right sided hyperplastic polyps will not become malignant. In a postmortem study conducted in New Zealand there were 43 right sided hyperplastic polyps among 333 subjects, or one hyperplastic polyp in eight subjects. ${ }^{38}$ One would expect to see one MSI-H colorectal cancer in approximately 200 subjects on the basis that the lifetime risk of developing colorectal cancer is approximately $5 \%$ but only $10 \%$ of sporadic colorectal cancers are MSI-H. ${ }^{39}$ Therefore, very approximately, only about one in 25 right sided hyperplastic polyps (dividing 200 by eight) might be expected to convert into an MSI-H cancer. This estimate does not include the non-MSI-H colorectal cancers with extensive DNA methylation that may also arise in hyperplastic polyps. ${ }^{40}$ These calculations are crude, but indicate that the removal of a relatively large flat lesion from the thin walled right colon in an elderly subject carries risks that may not necessarily be balanced by clinical benefit. Nevertheless, the malignant conversion rate for a right sided hyperplastic polyp is approximately the same as that for a colorectal adenoma.

In an unpublished series of 1436 colonoscopically derived polyps collected at the McGill University Health Centre (MUHC), Montreal, Canada, there were 51 right sided hyperplastic polyps and 251 left sided hyperplastic polyps, including the variant hyperplastic polyp described as sessile serrated adenoma. Twelve of the 51 right sided hyperplastic polyps and 18 of the 251 (7\%) left sided hyperplastic polyps showed the features of sessile serrated adenoma (table 1 ). Therefore, sessile serrated adenomas appear to show a predilection for the proximal colon when compared with classic hyperplastic polyps. Others report a somewhat higher frequency of sessile serrated adenomas in both the right and left colorectum, no doubt because of differing diagnostic thresholds. ${ }^{33}$ Although there is no direct evidence that left sided hyperplastic polyps confer an increased risk of malignancy, even when they show the features of sessile serrated adenoma, it cannot be assumed that hyperplastic polyps with malignant potential are restricted to the proximal colon. The polyps from the same MUHC series included 16 mixed polyps of various types (table 1). Among these were six left 
Table 1 Classification of 1436 polyps removed colonoscopically and consecutively at McGill University Health Centre, Montreal, Canada between 1992 and 1994

\begin{tabular}{lrrrrr}
\hline Diagnosis & RC & LC/R & Un site & Total & $\%$ \\
\hline TA & 186 & 402 & 125 & 713 & 50 \\
TVA & 38 & 128 & 33 & 199 & 14 \\
VA & 1 & 11 & 1 & 13 & 1 \\
HP & 39 & 233 & 30 & 302 & 21 \\
SSA & 12 & 18 & 1 & 31 & 2 \\
MP & 5 & 9 & 2 & 16 & 1 \\
SA & 1 & 16 & 1 & 18 & 1 \\
Other & & & & 144 & 10 \\
& & & & 1436 & 100 \\
\hline
\end{tabular}

$H P$, hyperplastic polyp; $L C / R$, left colon/rectum; $M P$, mixed polyp; Other, hamartomatous, inflammatory or unclassified; RC, right colon; SA, serrated adenoma; SSA, variant hyperplastic polyp or sessile serrated adenoma; TA, tubular adenoma; TVA, tubulovillous adenoma; Un site, unknown site; $V A$, villous adenoma.

Mixed polyps include: HP/SA (1), HP/TA (2), HP/TVA (1), SSA/SA (3), SSA/TA (2), SSA/VA (1), SA/TVA (4), SA/VA (2).

sided polyps that showed unequivocal adenoma arising within a hyperplastic polyp or sessile serrated adenoma. This illustrates the potential for left sided serrated polyps to undergo neoplastic evolution. Interestingly, of 18 pure serrated adenomas, only one was known to have arisen in the right colon (table 1). This suggests that right sided hyperplastic polyps (including the variant described as sessile serrated polyp) are biologically distinct from pure classic serrated adenomas.

\section{Take home messages}

- A subset of hyperplastic polyps has been linked with colorectal cancer showing DNA methylation and microsatellite instability

- Features of hyperplastic polyps with increased malignant potential include large size, multiplicity, location in the proximal colon, and a sessile growth pattern

- Hyperplastic polyps with malignant potential have been distinguished from classic hyperplastic polyps on the basis of subtle microscopic features that include increased serration, crypt dilatation, horizontal crypts, and hypermucinous epithelium

- Several diagnostic terms have been introduced for variant hyperplastic polyps, including "sessile serrated adenoma"

- A histological distinction between classic hyperplastic polyp and sessile serrated adenoma cannot be achieved in all cases

- Sessile serrated adenomas are characterised by frequent mutation of BRAF and DNA methylation, whereas traditional hyperplastic polyps are more likely to have K-ras mutation and less extensive DNA methylation

- Although most right sided hyperplastic polyps or sessile serrated adenomas will not progress to cancer, the magnitude of risk is probably comparable to that of traditional colorectal adenoma

- Clinicians should be made aware of the increased malignant potential of right sided hyperplastic polyps or sessile serrated adenomas
"Sessile serrated adenomas appear to show a predilection for the proximal colon when compared with classic hyperplastic polyps"

\section{A PERSONAL DIAGNOSTIC APPROACH}

The presence of a right sided hyperplastic polyp is recorded and attention is drawn to the increased potential of these lesions for future malignancy. The risk of future malignancy is likely to be higher in the case of right sided hyperplastic polyps that are unusually large, multiple, include dysplastic subclones (mixed polyp), have the features that have been associated with the variant hyperplastic polyp described as sessile serrated adenoma, or are associated with coexisting adenomas. Terms such as sessile serrated adenoma or sessile serrated polyp are not used in reports to the clinician. However, it is appropriate to highlight variant hyperplastic polyps with increased malignant potential in publications of basic and clinical research. Routine testing for MSI or for loss of DNA mismatch repair genes by immunohistochemistry is not undertaken because these alterations generally occur only with the advent of unequivocal dysplasia (intraepithelial neoplasia). ${ }^{4}$ It is possible that the establishment of deficient DNA mismatch repair results in rapid malignant evolution that would be analogous to the "aggressive adenomas" associated with hereditary non-polyposis colorectal cancer. ${ }^{41}$ Therefore, multiple slices through large hyperplastic polyps should be taken to exclude the presence of dysplasia. The new insights outlined above signal an important change in the management of serrated polyps of the colorectum.

\section{Authors' affiliations}

T Higuchi, Department of Digestive Surgery, Tokyo Medical and Dental University, Tokyo, Japan

J R Jass, Department of Pathology, McGill University, Montreal, Quebec, Canada H3A 2B4

\section{REFERENCES}

1 Jass JR, Young J, Leggett BA. Hyperplastic polyps and DNA microsatellite unstable cancers of the colorectum. Histopathology 2000;37:295-301.

2 lino H, Jass JR, Simms LA, et al. DNA microsatellite instability in hyperplastic polyps, serrated adenomas, and mixed polyps: a mild mutator pathway for colorectal cancer? J Clin Pathol 1999;52:5-9.

3 Mäkinen MJ, George SMC, Jernvall P, et al. Colorectal carcinoma associated with serrated adenoma-prevalence, histological features, and prognosis. J Pathol 2001;193:286-94.

4 Jass JR, lino H, Ruszkiewicz A, et al. Neoplastic progression occurs through mutator pathways in hyperplastic polyposis of the colorectum. Gut 2000;47:43-9.

5 Biemer-Hüttmann A-E, Walsh MD, McGuckin MA, et al. Mucin core protein expression in colorectal cancers with high levels of microsatellite instability indicates a novel pathway of morphogenesis. Clin Cancer Res 2000;6:1909-16.

6 Hawkins NJ, Ward RL. Sporadic colorectal cancers with microsatellite instability and their possible origin in hyperplastic polyps and serrated adenomas. J Natl Cancer Inst 2001;93:1307-13.

7 Day DW, Jass JR, Price AB, et al. Morson and Dawson's gastrointestinal pathology, 4th ed. Oxford: Blackwell, 2003.

8 Hamilton SR, Aaltonen LA. World Health Organisation classification of tumours. Pathology and genetics. Lyon: IARC Press, 2000.

9 Provenzale D, Garrett JW, Condon SE, et al. Risk for colon adenomas in patients with rectosigmoid hyperplastic polyps. Ann Intern Med 1990; 113:760-3.

10 Jass JR. Serrated route to colorectal cancer: back street or super highway? J Pathol 2001;193:283-5.

11 Longacre TA, Fenoglio-Preiser CM. Mixed hyperplastic adenomatous polyps/ serrated adenomas. A distinct form of colorectal neoplasia. Am J Surg Pathol 1990;14:524-37.

12 Ajioka $Y$, Watanabe $H$, Jass JR, et al. Infrequent $\mathrm{K}$-ras codon 12 mutation in serrated adenomas of human colorectum. Gut 1998;42:680-4.

13 Chan TL, Zhao W, Leung SY, et al. BRAF and KRAS mutations in colorectal hyperplastic polyps and serrated adenomas. Cancer Res 2003;63:4878-81.

14 Chan AO-O, Issa J-PJ, Morris JS, et al. Concordant CpG island methylation in hyperplastic polyposis. Am J Pathol 2002;160:529-36.

15 Park S-J, Rashid A, Lee J-H, et al. Frequent CpG island methylation in serrated adenomas of the colorectum. Am J Pathol 2003;162:815-22. 
16 Wynter CVA, Higuchi T, Leggett BA, et al. Methylation patterns define two types of hyperplastic polyp associated with colorectal cancer. Gut 2004;53:573-80.

17 Biemer-Hüttmann A-E, Walsh MD, McGuckin MA, et al. Immunohistochemical staining patterns of $\mathrm{MUC1}$, MUC2, MUC4, and MUC5AC mucins in hyperplastic polyps, serrated adenomas, and traditional adenomas of the colorectum. J Histochem Cytochem 1999;47:1039-47.

18 Jass JR, Whitehall VU, Young J, Leggett BA. Emerging concepts in colorectal neoplasia. Gastroenterology 2002;123:862-76.

19 Wang L, Cunningham JM, Winters JL, et al. BRAF mutations in colon cancer are not likely attributable to defective DNA mismatch repair. Cancer Res 2003:63:5209-12

20 Koinuma K, Shitoh K, Miyakura Y, et al. Mutations of BRAF are associated with extensive $\mathrm{hMLH} 1$ promoter methylation in sporadic colorectal carcinomas. Int J Cancer 2004; 108:237-42.

21 Jass JR. Serrated adenoma of the colorectum. Curr Diagn Pathol 2002;8:42-9.

22 Torlakovic E, Snover DC. Serrated adenomatous polyposis in humans. Gastroenterology 1996; 110:748-55.

23 Hayashi T, Yatani R, Apostol J, et al. Pathogenesis of hyperplastic polyps of the colon: a hypothesis based on ultrastructure and in vitro cell kinetics. Gastroenterology 1974;66:347-56.

24 Kaye GI, Fenoglio CM, Pascal RR, et al. Comparative electron microscopic features of normal, hyperplastic, and adenomatous human colonic epithelium. Variations in cellular structure relative to the process of epithelial differentiation. Gastroenterology 1973;64:926-45.

25 Frisch SM, Screaton RA. Anoikis mechanisms. Curr Opin Cell Biol 2001;13:555-62.

26 Cardone MH, Roy S, Stennicke HR, et al. Regulation of cell death protease caspase-9 by phosphorylation. Science 1998;282:1318-21.

27 Datta SR, Dudek H, Tao X, et al. Akt phosphorylation of BAD couples survival signals to the cell-intrinsic death machinery. Cell 1997:91:231-41.

28 Fenton RG, Hixon JA, Wright PW, et al. Inhibition of Fas (CD95) expression and Fas-mediated apoptosis by oncogenic Ras. Cancer Res 1998;58:3391-400.
29 Tateyama H, Li W, Takahashi E, et al. Apoptosis index and apoptosis-related antigen expression in serrated adenoma of the colorectum: the saw-toothed structure may be related to inhibition of apoptosis. Am J Surg Pathol 2002;26:249-56

30 Jass JR. Hyperplastic polyps and colorectal cancer: is there a link? Clin Gastroenterol Hepatol 2004;2:1-8.

31 Burt RW, Jass JR. Hyperplastic polyposis. In: Hamilton SR, Aaltonen LA, eds. World Health Organisation classification of tumours. Pathology and genetics. Tumours of the digestive system. Berlin: Springer-Verlag, 2000:135-6.

32 Frazier ML, Xi L, Zong J, et al. Association of the CpG island methylator phenotype with family history of cancer in patients with colorectal cancer. Cancer Res 2003;63:4805-8.

33 Torlakovic E, Skovlund E, Snover DC, et al. Morphologic reappraisal of serrated colorectal polyps. Am J Surg Pathol 2003;27:65-81.

34 Goldstein NS, Bhanot P, Odish E, et al. Hyperplastic-like colon polyps that preceded microsatellite unstable adenocarcinomas. Am J Clin Pathol 2003; 1 19:778-96.

35 Jass JR. Hyperplastic-like polyps as precursors of microsatellite unstable colorectal cancer. Am J Clin Pathol 2003:119:773-5.

36 Erhardt P, Schremser EJ, Cooper GM. B-Raf inhibits programmed cell death downstream of cytochrome $c$ release from mitochondria by activating MEK/ Erk pathway. Mol Cell Biol 1999; 19:5308-15.

37 Kambara T, Simms LA, Whitehall VU, et al. BRAF mutation and CpG island methylation: an alternative pathway to colorectal cancer. Gut 2004;53:[In press].

38 Jass JR, Young PJ, Robinson EM. Predictors of presence, multiplicity, size and dysplasia of colorectal adenomas. A necropsy study in New Zealand. Gut 1992;33:1508-14.

39 Ionov Y, Peinado MA, Malkhosyan S, et al. Ubiquitous somatic mutations in simple repeated sequences reveal a new mechanism for colonic carcinogenesis. Nature 1993;363:558-61.

40 Whitehall VLJ, Wynter CVA, Walsh MD, et al. Morphological and molecular heterogeneity within non-microsatellite instability-high colorectal cancer. Cancer Res 2002:62:6011-14

41 Jass JR, Stewart SM. Evolution of hereditary non-polyposis colorectal cancer. Gut 1992;33:783-6.

\section{$\mathrm{ECHO}$}

\section{COX-2 is strongest predictor of early death in malignant mesothelioma}

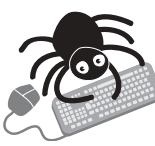

Please visit the Journal of Clinical

Pathology website [www. jclinpath.com] for a link to the full text of this article.
$\mathrm{N}$ ew findings may help to confirm how malignant mesothelioma develops, as cyclooxygenase-2 (COX-2) expression has been shown as a strong indicator of poor survival independently of other molecular markers previously thought to be involved. Only expression of COX-2 (hazard ratio 1.143), not of cell cycle inhibitors p21 and p27, was significantly associated with survival of patients with mesothelioma in multivariate analysis. Univariate analysis showed that high expression of COX-2 and low expression of p21 and p27 significantly reduced survival (from median 12-14 weeks to 5 weeks).Expression of p53 tumour suppressor protein was not significant in survival, possibly because the tumours were positive for SV40 sequences. COX-2 expression was not apparently related to tumour type-whether epithelioid, sarcomucoid, or of mixed histological type-all of which were represented.

The study cohort comprised 29 selected patients from 35 original patients available for follow up, all of whom were from one region in Italy and had mesotheliomas positive for SV40 virus sequences and p53 and p21 as determined previously. All patients had had surgery and 13 later had radiotherapy or chemotherapy. Expression of COX-2 and p27 was determined by histochemical staining of thin sections of biopsy specimens of the tumour with specific antibody. Patient survival was calculated from operation date.

The study confirms the role of COX-2 expression in determining survival in mesothelioma but is the first to investigate in vivo a potential relation with p53 and p21 and p27, which an earlier in vitro study had suggested might have an influence.

A Thorax 2004;59:428-433. 